\title{
Data-Driven Diabetes Education Guided by a Personalized Report for Patients on Insulin Pump Therapy
}

\author{
Danielle Groat ${ }^{1}$ Krystal Corrette ${ }^{2} \quad$ Adela Grando $^{2}$ Vaishak Vellore ${ }^{2}$ Mike Bayuk $^{2}$ George Karway ${ }^{2}$ \\ Mary Boyle ${ }^{3}$ Rozalina McCoy ${ }^{4}$ Kevin Grimm ${ }^{5}$ Bithika Thompson ${ }^{3}$
}

\footnotetext{
${ }^{1}$ Department of Biomedical Informatics, University of Utah School of Medicine, Salt Lake City, Utah, United States

${ }^{2}$ Department of Biomedical Informatics, Arizona State University, Tempe, Arizona, United States

${ }^{3}$ Department of Endocrinology, Mayo Clinic Arizona, Scottsdale,

Arizona, United States

${ }^{4}$ Division of Community Internal Medicine, Mayo Clinic, Rochester,

Minnesota, United States

${ }^{5}$ Department of Psychology, Arizona State University, Tempe,

Arizona, United States
}

ACl Open 2020;4:e9-e21.
Address for correspondence Danielle Groat, PhD, Department of Biomedical Informatics, University of Utah, 421 Wakara Way, \#140, Salt Lake City, UT 84108, United States (e-mail: dlgroat@asu.edu).

\section{Abstract}

\section{Keywords}

- type 1 diabetes

- phenotype

- smartphone

- diabetes mellitus

- self-care

- mhealth

- patients with chronic illness
Objective It is difficult to assess self-management behaviors (SMBs) and incorporate them into a personalized self-care plan. We aimed to develop and apply SMB phenotyping algorithms from data collected by diabetes devices and a mobile health (mHealth) application to create patient-specific SMBs reports to guide individualized interventions. Follow-up interventions aimed to understand patient's reasoning behind discovered SMB choices.

Methods This study deals with adults on continuous subcutaneous insulin infusion using a continuous glucose monitor (CGM) who self-tracked SMBs with an mHealth application for 1 month. Patient-generated data were quantified and an SMB report was designed and populated for each participant. A diabetes educator used the report to conduct personalized, data-driven educational interventions. Thematic analysis of the intervention was conducted. Results Twenty-two participants recorded 118 alcohol, 251 exercise, 2,661 meal events, and 1,900 photos. A patient-specific SMB report was created from this data and used to conduct the educational intervention. High variability of SMB was observed between patients. There was variability in the percentage of alcohol events accompanied by a blood glucose check, median $79 \%$ (38-100\% range), and frequency of changing the bolus waveform, median 11 (7-95 range). Interventions confirmed variability of SMBs. Main emerging themes from thematic analysis were: challenges and barriers, motivators, current SMB techniques, and future plans to improve glycemic control.

Conclusion The ability to quantify SMBs and understand patients' rationale may help improve diabetes self-care and related outcomes. This study describes our first steps in piloting a patient-specific diabetes educational intervention, as opposed to the current "one size fits all" approach. received

March 11, 2019 accepted after revision December 16, 2019

\author{
DOI https://doi.org/ \\ $10.1055 / \mathrm{s}-0039-1701022$. \\ ISSN 2566-9346.
}

(c) 2020 Georg Thieme Verlag KC Stuttgart . New York
License terms

(c) (i) 


\section{Background and Significance}

Type 1 diabetes mellitus (T1DM) is a chronic disease that requires consistent and iterative self-management with ongoing medical care, education, and support. ${ }^{1}$ Consistent glycemic control (e.g., avoidance of severe hypo- and hyperglycemia) is necessary to reduce the risk of immediate and long-term complications of poorly controlled diabetes. ${ }^{2-4}$ This is achieved by adherence to therapy and appropriate patientdirected adaptations that address planned and unplanned fluctuations invariably encountered in the real-world. Ensuring such adaptations is challenging due to the frequency and complexity of self-management behaviors (SMBs) related to blood glucose (BG) monitoring, insulin administration, food intake, physical activity, and use of increasingly complex diabetes technologies. Patients and health providers are further hindered by the inability to reliably capture, codify, and examine these SMBs, which preclude identification and correction of problems related to adherence or other aspects of disease management.

Self-reporting by means of surveys or interviews is one of the most widely used methods for evaluating SMBs and has been used in previous studies to understand SMBs for patients with T1DM. ${ }^{5-7}$ Assessing SMBs using self-reported data collected by means of surveys or interviews is simple and easy, and can provide useful information about SMBs. However, retrospective self-reporting can be subject to recall error and social desirability. ${ }^{6,8}$

Increasing availability and use of diabetes management technologies provide an opportunity to gather objective, real-time, and clinically meaningful information that can improve patient care. These include continuous subcutaneous insulin infusion (CSII) devices, or insulin pumps, which deliver insulin and have an embedded bolus calculator that can be personalized; glucose meters, which measure capillary glucose obtained by finger stick; and continuous glucose monitoring systems (CGMS), which measure interstitial glucose readings at regular intervals (e.g., 5 minutes). In addition to delivering insulin, CSII devices are capable of recording timestamps of delivered insulin, glucose levels (entered by patient or wirelessly uploaded from a compatible glucose meter and/or CGMS), carbohydrate intake (calculated and entered by patient), and patient interactions with the bolus calculator (interactive feature facilitating bolus insulin dosage).

Several studies have used glucose meter and/or CSII downloads to assess the frequency of BG monitoring, insulin bolus delivery, carbohydrate consumption, bolus calculator use, and meal-time bolus. ${ }^{9-12}$ While these studies used objectively measured data, the methods for quantifying adherence relied on manual and time-intensive extraction of information provided by vendor-specific reports from different device manufacturers. However, vendor-specific reports have been scrutinized for lacking actionable information for providers related to other SMBs that may influence glycemic control and fail to provide insight into what the patient's rationale was for the actions and adaptations taken. ${ }^{10,13-15}$ Vendor reports are also not generalizable across devices and technologies. Moreover, and partly as a result of these challenges, there are scarce data regarding adherence to CSII therapy and SMBs among adults with T1DM, as prior qualitative and quantitative studies have focused primarily on children or young adults. , $6,9-12,16-18^{-18}$

While recent evidence shows that there is considerable variability of SMBs within and between adult patients with T1DM on insulin pump therapy who use CSII, ${ }^{19-22}$ current behavioral interventions are mostly based on the "one size fits all" approach of standardized diabetes self-management education. ${ }^{1}$ This is unfortunate since individuals are more likely to follow self-management protocols that take their lifestyle preferences into account. ${ }^{23}$ The absence of personalized diabetes education is driven, in part, by the lack of objective and easily interpretable information regarding patient behavior outside the confines of the clinical encounter. The authors hypothesize that SMB information derived from self-tracked and device data could guide providers during the delivery of patient-specific diabetes educational interventions. This study describes our initial work in testing the feasibility of our methods.

\section{Objectives}

We sought to develop and apply computable SMB phenotyping algorithms capable of integrating and analyzing CSII and CGMS data with real-time patient-reported data collected with a mobile health (mHealth) application to quantify SMBs with high temporal fidelity. The second goal was to create an individualized SMB report template (user interface), and provide actionable information which could then be used by health care providers to facilitate individualized patient education, counseling, and treatment modification. We then aimed to discover patient's reasoning behind discovered SMBs through the thematic analysis of the personalized educational interventions.

\section{Methods}

-Fig. 1 provides an overview of the study design. Below we describe in detail each step of the study.

\section{Design of the Report Template}

Previous research by the study team examined and characterized SMBs in adults of all ages with T1D. ${ }^{19-22,24}$ Building on knowledge gleaned from that work, SMBs identified and quantified by the biomedical informatics team and a statistician were presented to a diabetes care team for review. An iterative process with feedback from all members of the research team was used to refine the report templates to present the behaviors in a meaningful format for health providers at the individual patient level.

Eleven SMBs ( - Table 1) were included in the behavioral report. Although frequency of hypoglycemic events (HypoFreq) is not a behavior, it was included due to the importance of limiting hypoglycemia when engaging in self-care. For behaviors alcohol, exercise, and meal compensation within 30 minutes (Alc30, Exer30, and Meal30, respectively) desired compensations were: check BG, consume and/or input carbs, deliver insulin bolus, and adjust basal rate. 


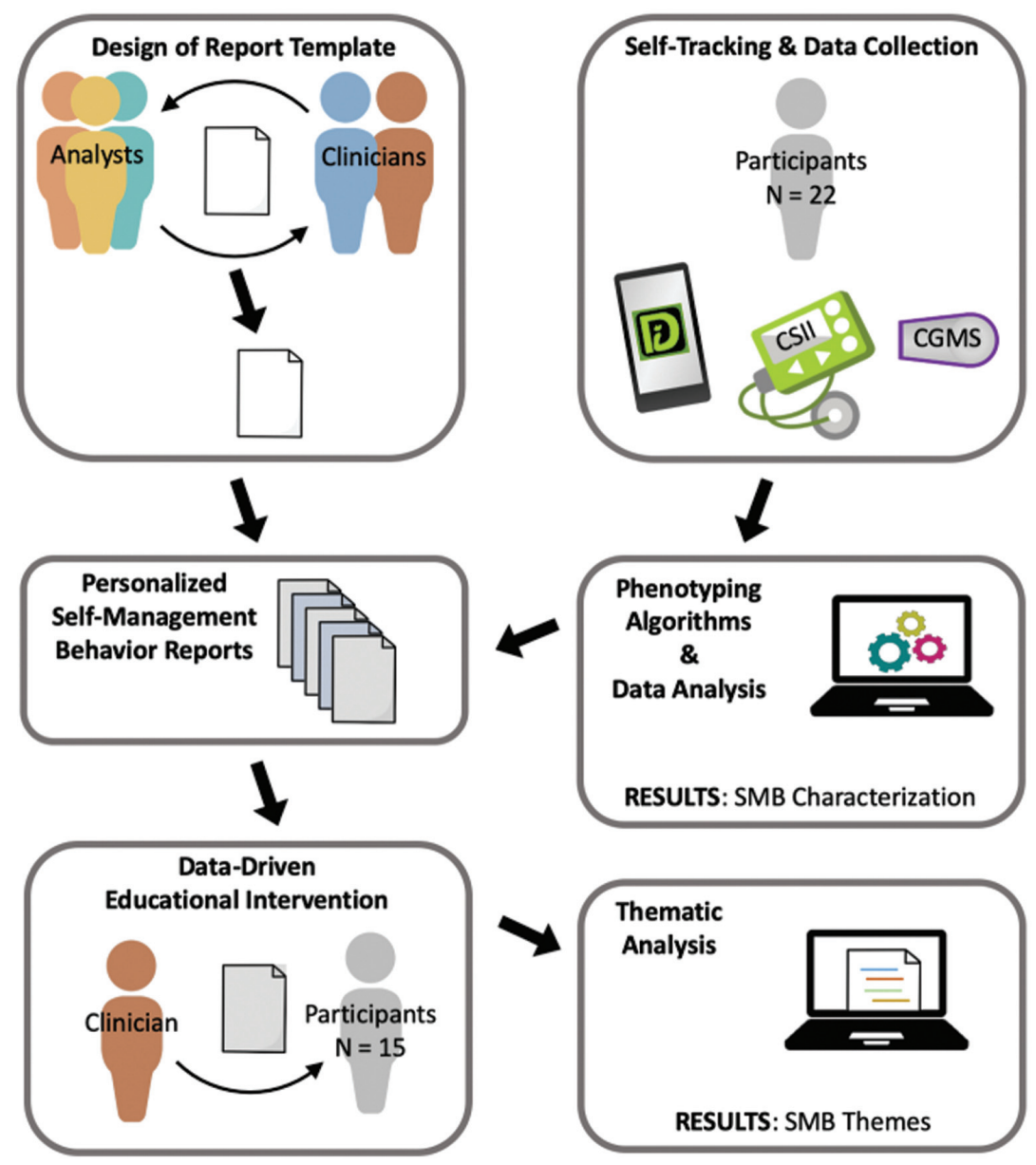

Fig. 1 Overview of study design to design and deliver data-driven educational interventions to participants with type 1 diabetes. Data that populated the personalized behavior reports were collected from continuous subcutaneous insulin injection (CSII) devices and continuous glucose monitoring systems (CGMS) while participants self-tracked with the iDECIDE app for 1 month. The outcomes of the data analysis were SMB characterizations. During in-person, one-to-one interviews a clinician met with each study participant to discuss the resulting personalized behavior report. The outcomes of the interviews were summarized as SMB themes. SMB, self-management behavior.

\section{Subject Recruitment, Self-Tracking, and Data Collection}

Adults with T1DM were recruited from an outpatient endocrinology clinic which provides consultative and longitudinal care to patients with T1DM. Institutional Review Board (IRB) approval from Mayo Clinic Arizona was granted to recruit subjects between the ages of 18 through 70 years, who spoke English, and owned an iPhone. Participants were recruited from January through May of 2018. Subjects were required to have been under the care of the clinic and had a Medtronic CSII device and any brand of CGMS for at least 1 year. We included patients using the Medtronic 670G pump, which can be configured as a hybrid-closed-loop system (auto mode), as well as those who used a traditional Medtronic pump (manual mode). The study was limited to Medtronic CSII devices to simplify algorithm development while capturing the largest number of patients. Participants were compensated during the study.

After recruitment, participants provided informed consent. Next, the iOS mHealth app, iDECIDE, was installed on the participants' smartphones and they were taught how to use the app to self-track exercise, meal, and alcohol intake. ${ }^{24}$
Participants were instructed to otherwise maintain their normal self-care routine for 1 month. When tracking exercise (ExerFreq), the app required that users provide the start time, duration in minutes, and intensity rating as light, moderate, or vigorous using the "talk test" as a guide ( - Fig. 2A). ${ }^{25}$ They were then asked to select from a menu of compensatory options: basal adjustment, my bolus choice, pump's bolus suggestion, pump disconnect, snack intake, other technique, or no compensation (Exer30) (-Fig. 2B). When logging meals and alcoholic beverages (AlcFreq), users were prompted to indicate the time of intake, provide a text description, and log the grams of carbohydrates for each item, with an option to add a photo before saving (AlcExmp, MealExmp; - Fig. 2C). They were then asked to select from a list of compensatory techniques used to manage glucose levels. For meals, users selected from: basal adjustment, my bolus choice, pump's bolus selection, square delivery adjustment, other technique, and no compensation (Meal30, ChgW; -Fig. 2D). Similarly, for alcoholic products, options included: meal consumption, basal adjustment, my bolus choice, pump's bolus suggestion, square delivery adjustment, other technique, and no compensation (Alc30, ChgW; -Fig. 2E). For exercise, meals, and alcohol, participants were 
Table 1 An overview of the data sources used to quantify behaviors. "A" indicates an automatic analysis by the phenotyping algorithms prior to manually populating the behavioral report template, while " $\mathrm{M}$ " indicates a manual review was used

\begin{tabular}{|c|c|c|c|c|}
\hline Self-management behavior & Explanation & $\begin{array}{l}\text { mHealth } \\
\text { app }\end{array}$ & $\begin{array}{l}\text { Insulin } \\
\text { pump }\end{array}$ & CGMS \\
\hline 1. Alcohol compensation \pm 30 min. (Alc30) & Compensate for alcohol within $30 \mathrm{~min}$ & A & A & \\
\hline 2. Exercise compensation \pm 30 min. (Exer30) & Compensate for exercise within $30 \mathrm{~min}$ & A & A & \\
\hline 3. Meal compensation \pm 30 min. (Meal30) & Compensate for meals within 30 min & A & A & \\
\hline 4. Change waveform (ChgW) & $\begin{array}{l}\text { Change insulin delivery from normal waveform } \\
\text { (i.e., standard insulin delivery mode) to dual or } \\
\text { square (i.e., time-extended delivery of insulin) }\end{array}$ & & A & \\
\hline 5. Bolus more than 10 times per day (Bol10) & Bolus 10 or more times per day & & A & \\
\hline 6. Days with low glucose (HypoFreq) & $\begin{array}{l}\text { Frequency of days with low BG } \\
\text { (hypoglycemia defined as }<70 \mathrm{mg} / \mathrm{dL} \text { ) }\end{array}$ & M & M & A \\
\hline 7. Exercise frequency (ExerFreq) & Frequency of exercise & A & & \\
\hline 8. Alcohol frequency (AlcFreq) & Frequency of alcohol consumption & A & & \\
\hline 9. Example of alcohol (AlcExmp) & $\begin{array}{l}\text { Patient-provided examples of alcoholic } \\
\text { beverages consumed and } \\
\text { carbohydrate estimates }\end{array}$ & M & & \\
\hline 10. Example of meal (MealExmp) & $\begin{array}{l}\text { Patient-provided examples of meals consumed } \\
\text { and carbohydrate estimates }\end{array}$ & M & & \\
\hline 11. Auto/manual mode (AvsM) & $\begin{array}{l}\text { Time spent in auto-mode versus manual mode } \\
\text { (only for } 670 \mathrm{G} \text { Medtronic insulin pump). }\end{array}$ & & M & \\
\hline
\end{tabular}

Abbreviations: BG, blood glucose; CGMS, continuous glucose monitoring systems.

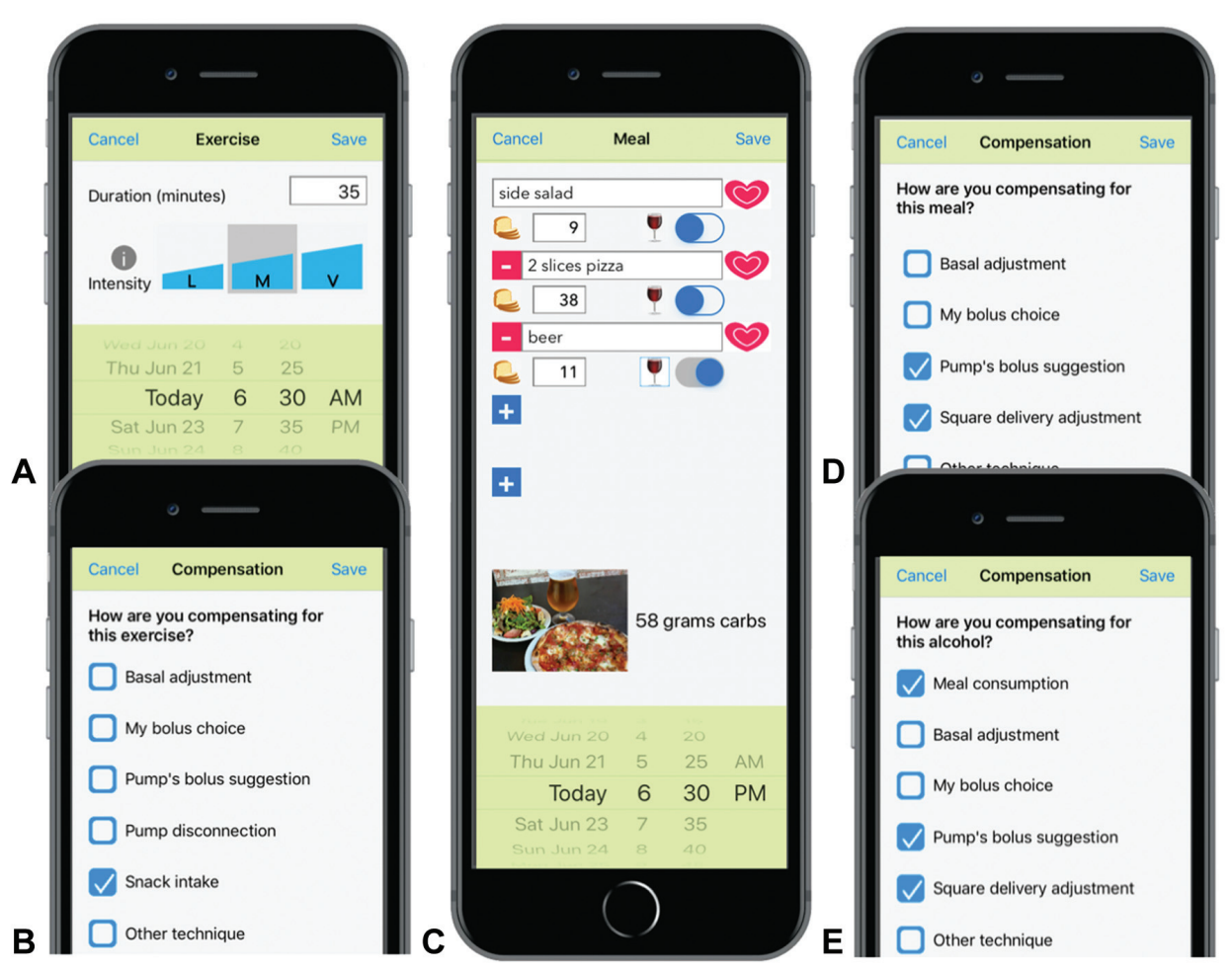

Fig. 2 Screenshots of the mHealth application, iDECIDE, that was installed on each subject's smartphone. Participants could self-track exercise by indicating (A) duration, intensity, start time, and (B) compensation techniques used to self-manage glucose. Meals and alcohol were selftracked with the same interface, by providing (C) text description of meal or alcohol, carbohydrate count, while also attaching a photo to the log and indicating the time. Participants were then prompted to select (D) meal, and (E) alcohol compensation techniques. 
allowed to choose more than one compensation technique, if applicable.

Subject's app data were stored locally on the smartphone and synced to a cloud service. Using password protected administrative privileges, members of the research team downloaded data from the cloud in spreadsheet format. At the end of the study period, subjects synced their CSII and CGMS data to the manufacturer web portals. The research team was then able to download the device data in spreadsheet form. Reports generated by the CSII manufacturer's web portals and demographics from the medical records were also accessed as part of the study. Participant age, years since diabetes diagnosis, years of CSII usage, and most recent glycosylated hemoglobin (HbA1c) results were obtained from the medical record.

\section{Phenotyping Algorithms and Data Analysis}

Phenotyping algorithms were developed in Java version 8 to accommodate the data format of each data source. Modifications to the algorithms were required to enable execution against the latest CSII model, the Medtronic 670G. The phenotyping algorithms produced output in spreadsheet format for each participant, which allowed for individual analyses and populating the reports. The individual-specific spreadsheets were consolidated to facilitate cohort level analyses. The number of days of data from each participant were calculated based on days where self-tracking data and device data were both present. Excel formulas were used to generate descriptive statistics and characterize the SMBs at the individual and cohort level. All data are reported as mean (standard deviation) or median (interquartile range).

For behaviors Alc30, Exer30, Meal30, ExerFreq, and AlcFreq, the log event of interest (e.g., alcohol, exercise, or meal) and CSII data were both read by the phenotyping algorithm. The timestamp of each log event was used as a starting point to quantify the days where exercise occurred, or meal/alcohol was consumed, and identify frequency and interactions of interest (e.g., check BG, consume/input carbohydrates, deliver insulin bolus, or basal adjustment) recorded by the CSII that occurred within 30 minutes prior, during, or after the event of interest. Use of the 30-minute window accounted for the fact that patients with T1DM may engage in compensatory behavior in anticipation of events or react to them after the fact. Also, patients often record events rounded to the nearest 5,10 , or 15 -minute intervals such that there may be slight variation in recorded versus actual event times.

CSII data were used to quantify ChgW, Bol10, and AvsM, while CGMS was used to determine HypoFreq. For behaviors ChgW and Bol10, the CSII data were read by the algorithm to detect all instances of delivered boluses. Insulin boluses delivered each day were counted, and days with $\geq 10$ boluses were flagged and counted. Additionally, each bolus waveform was classified as normal or extended (i.e., dual or square waveform), and each day where one or more of the boluses was changed to extended was counted. For HypoFreq, glucose readings were scanned, and days with one or more glucose values $<70 \mathrm{mg} / \mathrm{dL}$ were flagged and counted. The amount of time in auto-mode, AvsM, was obtained from the manufacturer's patient report (specific to the Medtronic 670G).

\section{Personalized SMB Report}

The personalized SMB report templates were populated by manually inserting the desired data points from the phenotyping algorithm results into the appropriate fields of the report for each participant. The exception was AvsM and portions of Bol10 and HypoFreq. Screenshots from the vendor reports were used to populate day-specific information for Bol10 and HypoFreq. Metrics for AvsM were obtained from the vendor report. - Fig. 3 shows the first page of a sample report reviewing all of the observed behaviors. Behaviors Alc30, Exer30, Meal30, ChgW, Bol10, and HypoFreq were color coded as red, yellow, or green to highlight SMBs that could benefit from further discussion during in-person patient interventions. For instance, a low frequency of use of extended bolus was highlighted as red to guide discussions on the use of this advance feature of the CSII. - Fig. 4 depicts the second page of the report, which provides more details on HypoFreq and the events such as food/alcohol intake and exercise surrounding any day with low glucose.

\section{Data-Driven Educational Intervention}

Participants were each re-contacted individually and invited to participate in a personalized data-driven educational intervention. IRB approval from Mayo Clinic was granted for the intervention portion of the study. Participants were recruited and re-consented from May through September, 2018 and compensated for their participation. Prior to meeting with the participants, the endocrinologist and the nurse practitioner diabetes educator reviewed and discussed the personalized SMB report (M.B. and B.T., respectively). The diabetes educator then met with the participant and used the SMB report to guide a semistructured, personalized educational intervention. The intervention was attended by a member of the research team (K.C.) and audio-recorded.

The intervention started with the diabetes educator sharing the SMB report with the participant and explaining that the color coding was only used to indicate areas that require more discussion time for the provider to get a better understanding of the participant's lifestyle and preferences. The remainder of the intervention was semi-structured and followed a script that was adapted according to the participant's report. For example, if the report indicated that the participant consumed alcohol during the study, section 2 of the interview script was included in the intervention and the participant was provided a handout on alcohol and diabetes, otherwise section 2 and the accompanying handout was omitted from the intervention.

\section{Thematic Analysis of Intervention}

The audio-recording of the provider delivering the intervention to the participant was transcribed by a commercial third-party company. The contents of the audio-recordings and transcriptions were then analyzed using the six phases of Braun and Clarke's thematic analysis guidelines. ${ }^{26}$ First, two members of the research team independently reviewed the audio-recordings to gain an overview. Three initial transcripts were separately coded, and preliminary codes were assigned to the data extracts with the intent of coding for as many potentially interesting themes as possible. A third 


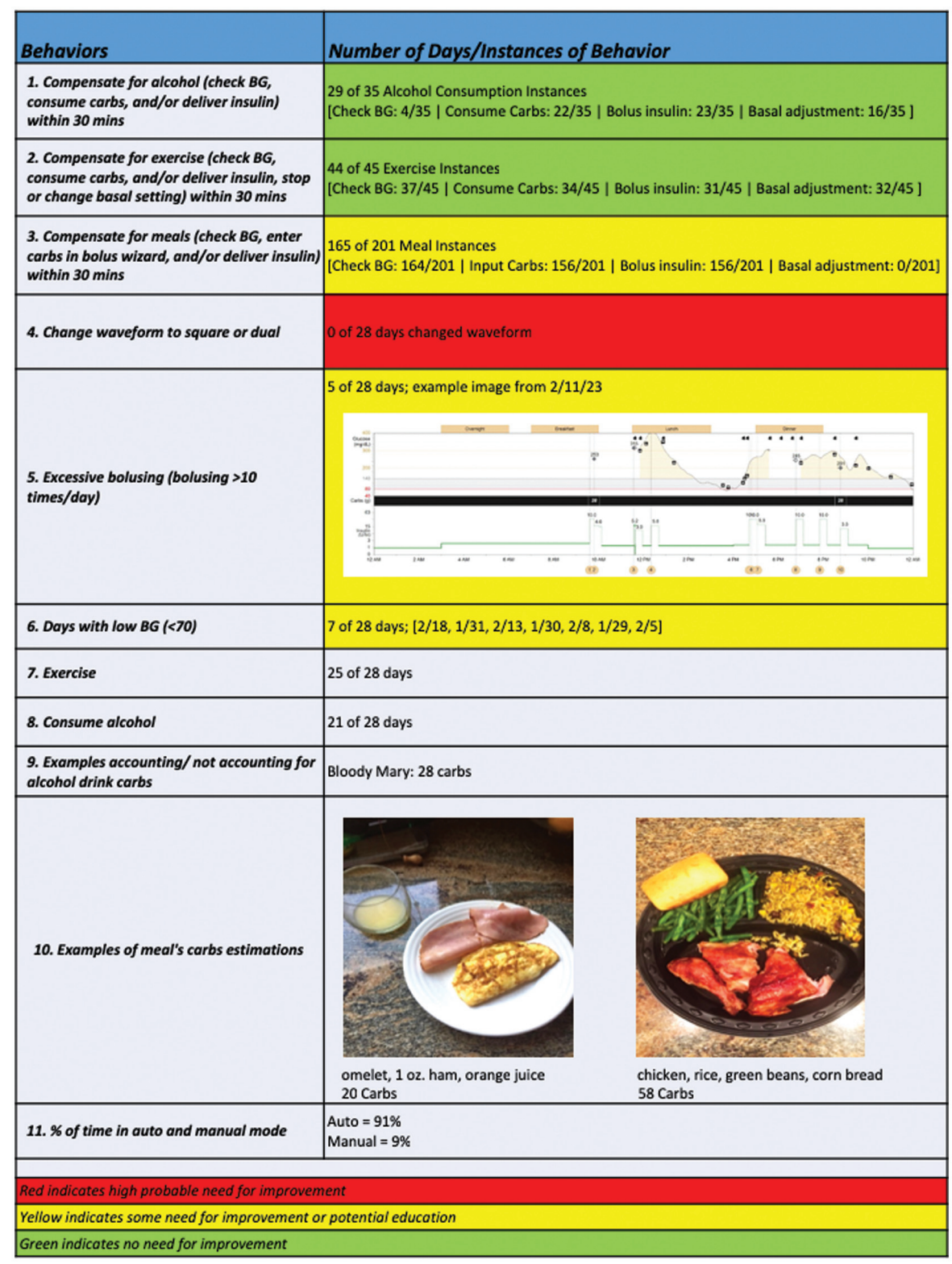

Fig. 3 Example of the first page of the behavioral report template used to facilitate the personalized educational intervention phase of the study. Behaviors 1 to 6 were color coded as red, yellow, and green to guide providers in the review of data and the organization of the interventions. Behaviors in red indicated that an in-depth discussion was recommended to better contextualize the specific behavior in terms of patient's lifestyle choices. Yellow indicated that some contextualization could be needed to help interpret the behaviors. Green indicated little need for in-depth discussion.

researcher helped to resolve disagreements to reach consensus. Cohen's Kappa was used to determine coder agreement. In an iterative process, themes were identified, reviewed, and refined across interviews by the research team. MAXQDA 12.3.5 was used to code the transcripts.

\section{Results}

\section{Demographics}

Twenty-two subjects were recruited for the self-tracking portion of the study. Mean age was 48.1(16.7) years with a mean duration of T1DM 29.5(18.5) years and CSII use 15.6 (8.4) years. All subjects were Caucasian and 15 were female. Mean $\mathrm{HbA} 1 \mathrm{c}$ was $7.0 \%(0.86 \%)$ and the percentage of CGMS readings at target was $48.8 \%(12.0 \%) ; 48.1 \%(13.6 \%)$ of the CGMS readings were above target and $3.2 \%(3.5 \%)$ were below target. Insulin pump models used by the subjects were: Paradigm $523(n=1)$, Paradigm 630G $(n=1)$, Paradigm 530G $(n=4)$, Paradigm $751(n=4)$, and Paradigm 670G $(n=12)$. CGMS models used were: Medtronic Enlite $(n=7)$, Dexcom G5 $(n=7)$, and Medtronic Guardian $(n=8)$.

\section{Characterization of Self-Management Behaviors}

\section{Data Collection}

A total of 267,083 data points were collected by CSII and CGMS spanning 604 patient-days. Using the mHealth app, participants logged 118 alcohol events, 251 exercise events, 


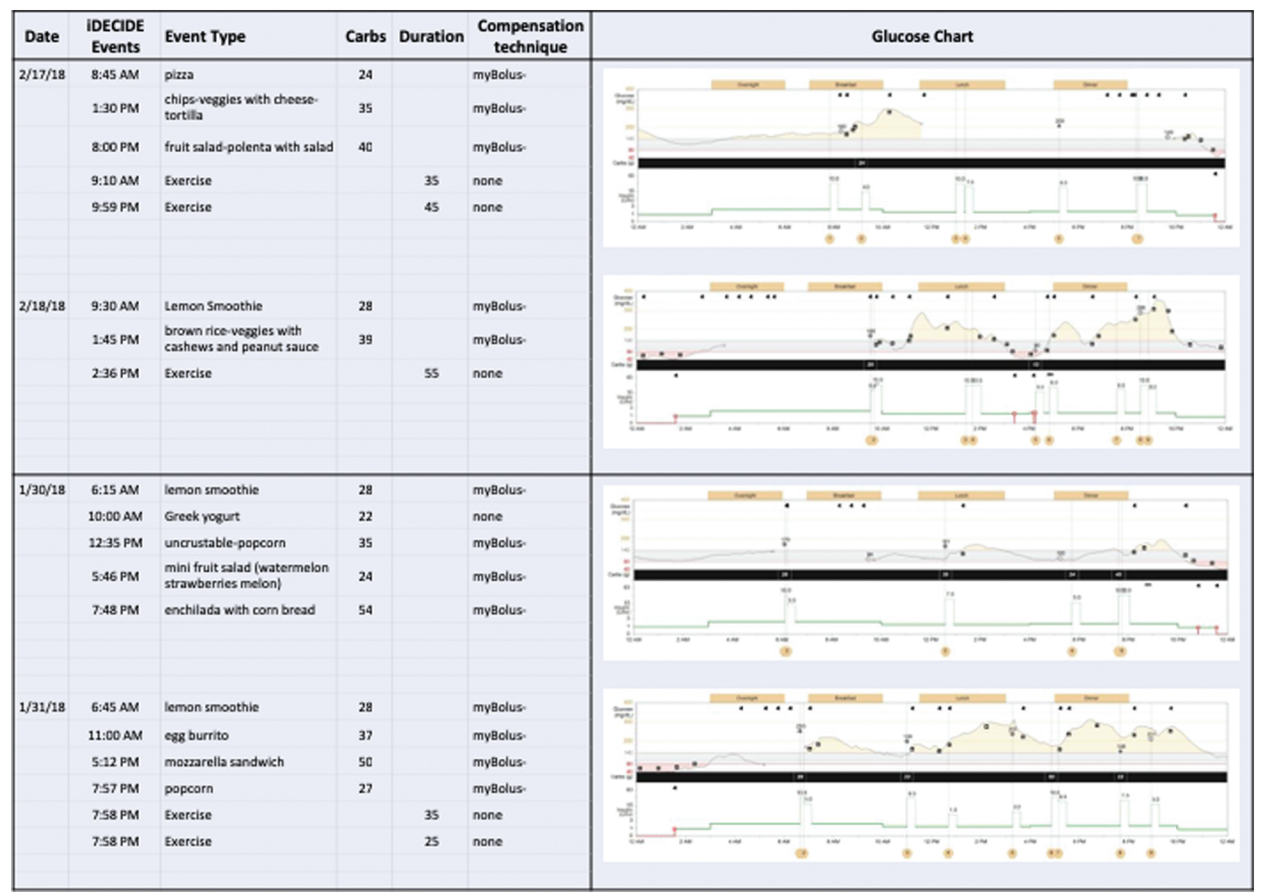

Fig. 4 Example of the second page of the behavioral report template highlighting days where hypoglycemia occurred during the self-tracking portion of the study.

2,661 meals, and 1,900 meal photos. The mean number of days with complete data (i.e., complete data coverage from the convergence of CSII, CGMS, and mHealth app) was 27.5 (2.5). This resulted in 5.4(9.7) alcohol events, 11.4(12.1) exercise events, 120.1(51.0) meals, and 86.4(51.2) meal photos per participant.

\section{Alcohol, Exercise, and Meals}

Thirteen participants reported alcohol consumption on the mHealth app, with an average of 9.1(11.2) alcohol logs (AlcFreq). Based on CSII data, participants who reported alcohol consumption were observed to have the greatest variability in three of four SMBs of interest as seen in - Fig. 5A (Alc30). Participants compensated for alcohol by consuming carbohydrates in conjunction with alcohol in $100 \%(63-100 \%$ ) of events, delivering an insulin bolus in $100 \%$ (64-100\%), checking BG in 79\%(38-100\%), and adjusting the basal rate in $29 \%(0-88 \%)$.

Eighteen participants engaged in exercise during the study; they tracked an average of 13.9(12.1) exercise episodes (ExerFreq). There was considerable variability of SMBs observed in the CSII data of those who engaged in exercise ( - Fig. 5A). Participants engaged in carbohydrate consumption in $67 \%(50-77 \%)$ of the events, insulin bolus delivery in $68 \%(50-81 \%)$, checking BG in 67\%(43-75\%), and basal rate adjustment in 34\%(0-62\%) (Exer30).

All participants self-tracked meals during the study. Meals self-tracked with the mHealth app were accompanied by a corresponding user-entry in the CSII bolus calculator in $66 \%(53-78 \%)$ of the events. There was less variability in SMBs for meals when compared with alcohol and exercise in corresponding CSII data ( $\rightarrow$ Fig. 5A). Participants delivered insulin in $68 \%(47-81 \%)$ of the events, checked BG in $54 \%$
(40-81\%), and adjusted the basal rate in $18 \%(0-29 \%)$, as can be seen in - Fig. 5A (Meal30).

\section{Insulin Pump Interactions}

Eight participants changed the insulin bolus waveform from normal to extended on at least one or more occasions. Overall, participants engaged in changing the waveform $0(0-9)$ times during the study (ChgW), but when considering those that changed the waveform one or more times, the frequency changed to $11(7-95)$. Thirteen participants bolused $\geq 10$ times/d on at least one or more days, with a cohort median of frequent bolusing of $1(0-5)$ days, ( - Fig. $5 B$; Bol10).

There were 12 participants using the Medtronic 670G insulin pump. One participant never used the auto-mode feature, while another used the auto-mode feature for the entire duration of the study. On average, the 12 participants were in auto-mode $87 \%(59-94 \%)$ of the time during the study, (-Fig. 5B; AvsM).

\section{Hypoglycemia}

All participants had at least 3 days where hypoglycemia (i.e., one or more CGMS readings $<70 \mathrm{mg} / \mathrm{dL}$ ) occurred, with a median of 12(7-19) out of an average 27.5(2.5) days, (-Fig. 5B; HypoFreq).

\section{Self-Management Behavior Themes}

\section{Demographics}

Fifteen individuals participated in the data-driven educational intervention. Mean age was 47(15.7) years with a mean duration of T1DM 33.7(17.7) years of CSII use 16.7(8.8) years. All were Caucasian and nine were female with a mean HbA1c $7.0 \%(0.44 \%)$. 

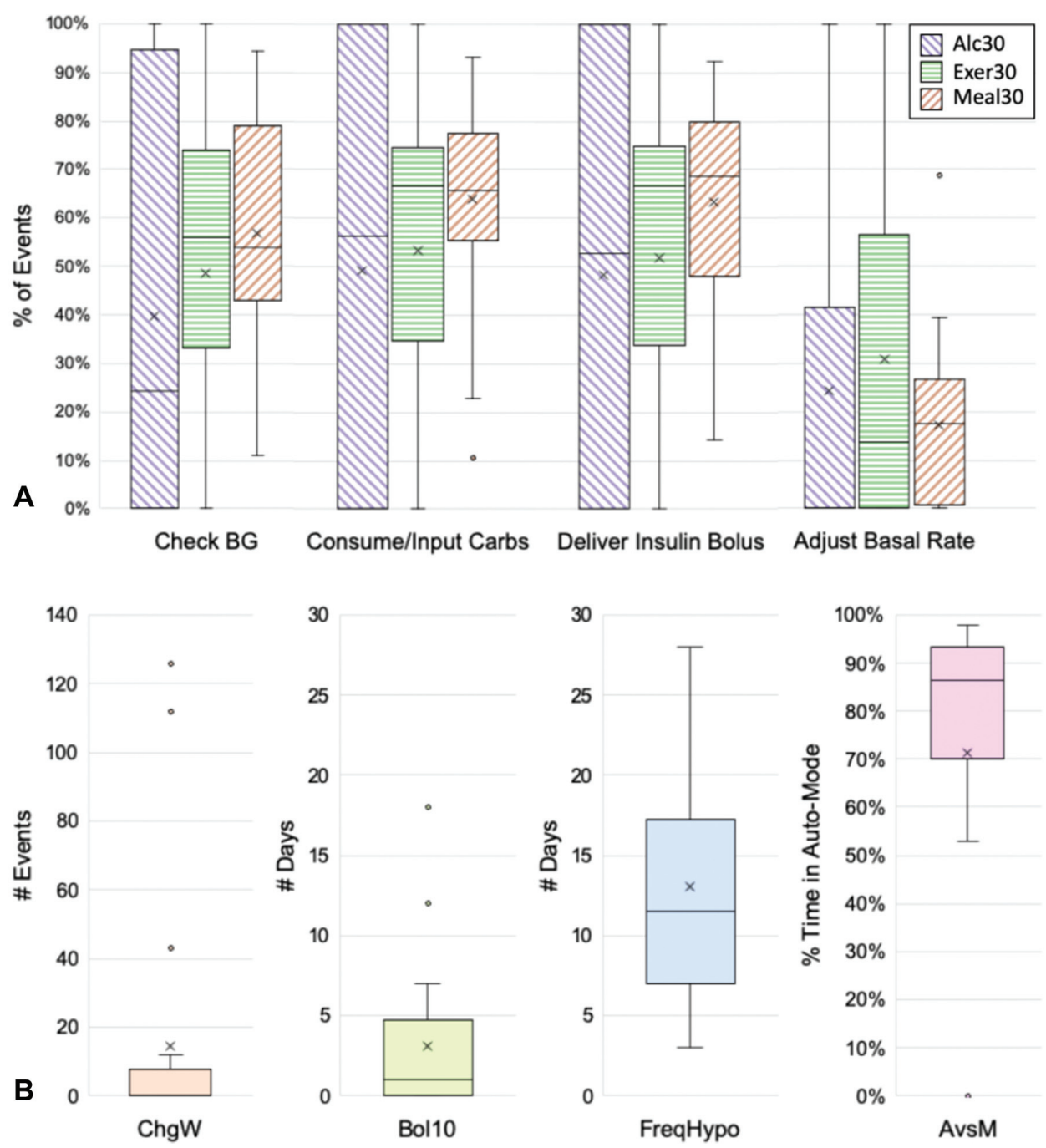

Fig. 5 Variability of self-management behaviors in 22 participants was observed in (A) the percentage of events that participants compensated for alcohol (Alc30), exercise (Exer30), and meals (Meal30) within 30 minutes of the event, and for (B) insulin pump interactions, such as changing the insulin bolus waveform (ChgW), delivering 10 or more insulin boluses a day (Bol10), the number of days that hypoglycemia occurred (FreqHypo), and the percentage of time those with the 670G hybrid-closed loop pump used the automatic-mode instead of the manual-mode (AvsM).

\section{Theme Analysis}

Thematic analysis identified four key themes related to diabetes self-management: challenges and barriers ( - Table 2), motivators (-Table 3), current SMBs and Practices (-Table 4) and future changes and plans based on the personalized datadriven behavior report ( - Table 5). For each theme, subthemes were identified. - Tables 2-4 to 5 list the subthemes, subtheme definitions, number $(n)$, and percentage (\%) of participants for each subtheme, and an illustrative quote of each subtheme. Cohen's kappa was 0.96 , indicating nearly complete interrater agreement.

In terms of self-management challenges and barriers $(-$ Table 2 ), there were notable variations in the barriers to healthy living habits that participants mentioned. These included medical conditions such as coronary conditions, foot and knee problems, gallbladder surgery, celiac disease, longterm back pain, and depression as well as stress and lack of time (12 hours work shifts and taking care of a toddler). Dissatisfaction with technology was another barrier mentioned. Main sources of dissatisfaction were the bolus wizard, sensor accu- racy and calibration, auto-mode functionality (670G users), and general dissatisfaction with diabetes technology. ${ }^{27}$ Another barrier identified is the perception that "rules do not apply to me." When conducting educational interviews, there were seven participants that identified themselves as "medical outliers," implying that common, evidence-based educational recommendations would not work or did not apply to them. For example, one participant felt that published expert recommendations regarding continuous glucose monitor (CGM) trend arrows (for example, how to decide if more insulin is needed depending on the trend and arrow directionality on CGM) did not apply to her and would bottom her out. When discussing common patterns of insulin resistance during certain times of the day, one participant stated "my body behaves just the opposite of that." Multiple participants felt the "rule of 15 ," a commonly used method to recommend intake of $15 \mathrm{~g}$ of carbohydrate to fix a low blood sugar followed by waiting 15 minutes to recheck the sugar, would not work for them.

In terms of motivators ( - Table 3 ), avoiding hypoglycemic events during the night or day was an important one. 
Table 2 Challenges and barriers to diabetes self-management $(n=15)$

\begin{tabular}{|c|c|c|c|}
\hline Theme & Definition & $n(\%)$ & Quote \\
\hline $\begin{array}{l}\text { Barriers to healthy } \\
\text { living habits }\end{array}$ & $\begin{array}{l}\text { Permanent or temporal factors that are } \\
\text { outside control and affect ability to } \\
\text { self-manage blood glucose. Includes other } \\
\text { medical conditions. }\end{array}$ & $10(67)$ & $\begin{array}{l}\text { "When you have a toddler }[. . .] \text { you've got } \\
\text { your hands busy and eating on time is } \\
\text { difficult." }\end{array}$ \\
\hline $\begin{array}{l}\text { Management of } \\
\text { overnight hypoglycemia }\end{array}$ & $\begin{array}{l}\text { Overnight hypoglycemia incidents that are } \\
\text { hard to manage and may cause sleep } \\
\text { disruptions or dangerous lows. }\end{array}$ & $10(67)$ & $\begin{array}{l}\text { "Because you are so low and you just feel } \\
\text { so out of control that you just eat and eat, } \\
\text { and eat, and eat." }\end{array}$ \\
\hline Unpredictable variations & $\begin{array}{l}\text { Unpredictable/unexplainable fluctuations } \\
\text { in blood glucose levels that may cause } \\
\text { frustration due to lack of control. }\end{array}$ & $8(53)$ & $\begin{array}{l}\text { "One day things seem to go perfect, the } \\
\text { next day I can eat the exact same thing and } \\
\text { it's not even close." }\end{array}$ \\
\hline $\begin{array}{l}\text { Dissatisfaction } \\
\text { with technology }\end{array}$ & $\begin{array}{l}\text { Distrust in the accuracy of technology, or } \\
\text { dissatisfaction with available technology. } \\
\text { Hesitation to rely solely on technology. } \\
\text { Comments on suspected technology } \\
\text { dysfunction. }\end{array}$ & $8(53)$ & $\begin{array}{l}\text { "I didn't use the auto-mode for very long, } \\
\text { but I had tried doing a temp blood sugar } \\
\text { and it didn't work. I was still going low." } \\
\text {-Medtronic 670G user }\end{array}$ \\
\hline $\begin{array}{l}\text { "Rules don't apply } \\
\text { to me” }\end{array}$ & $\begin{array}{l}\text { Perception that the individual is an outlier } \\
\text { or their blood glucose levels do not } \\
\text { respond in a predictable manner according } \\
\text { to evidence based guidelines. }\end{array}$ & $7(47)$ & $\begin{array}{l}\text { "I'm a medical outlier. That's just all there } \\
\text { is to it. I don't follow the usual statistics." }\end{array}$ \\
\hline Human error & $\begin{array}{l}\text { Mistakes made by the participant in } \\
\text { managing blood glucose such as } \\
\text { forgetting to bolus or other errors. }\end{array}$ & $6(40)$ & $\begin{array}{l}\text { "That may happen once every } 2 \text { or } 3 \text { mo } \\
\text { that I forget to bolus." }\end{array}$ \\
\hline Insurance coverage & $\begin{array}{l}\text { Mention of insurance constraints and lack } \\
\text { of coverage. Includes cost of insurance and } \\
\text { cost of supplies or technology. }\end{array}$ & $3(20)$ & $\begin{array}{l}\text { "My insurance stopped covering the strips } \\
\text { for [. . . ] Minimed's meter." }\end{array}$ \\
\hline
\end{tabular}

Participants mentioned traumatizing experiences involving passing out in public places and need for emergency paramedic assistance, as well as endangering the life of family members when passing out and causing a car accident. In addition, participants were motivated to avoid chronic health complications, in particular avoidance of heart, brain, feet, and eye complications.

When discussing current SMBs used ( - Table 4), six participants mentioned relying strongly on the pump and bolus wizard, while the other seven participants mentioned the value of the CGM to achieve glycemic control: "the continuous monitor has been probably the best tool so far." There was considerable variation in the type of SMBs mentioned to compensate for exercise, including eating before exercise to avoid lows, bolusing before/after exercise to avoid highs, checking BG before exercise, taking off the pump during exercise, and lowering or suspending the basal rate during exercise. All the participants were aware of the importance of checking trend arrow directions, but not all of them consistently took the arrows into account when making SMB decisions. Participants mentioned difficulty assessing food volume, content and fat, particularly when eating out. Some participants indicated preferring to eat at home, weighing everything they ate, or following a routine of eating the same foods to help with meal compensations. As part of the support network, participants mentioned the active involvement of family and health professionals. One participant used technology (CGM sends low BG alerts to designated people) as a

Table 3 Motivators of diabetes self-management $(n=15)$

\begin{tabular}{|l|l|l|l|}
\hline Theme & Definition & $n(\%)$ & Quote \\
\hline $\begin{array}{l}\text { Avoid hypoglycemia or } \\
\text { hyperglycemic events }\end{array}$ & Fear of hypo/hyperglycemia & $4(27)$ & $\begin{array}{l}\text { "That [hypoglycemia] was particularly } \\
\text { problematic [. .] I experience more lows } \\
\text { than what statistically is healthy." }\end{array}$ \\
\hline Avoid health complications & $\begin{array}{l}\text { Fear of health } \\
\text { complications/comorbidities }\end{array}$ & $3(20)$ & $\begin{array}{l}\text { “...That stuff has got to change and why has } \\
\text { it got to change, because now, I have } \\
\text { bleeds in the back of my eyes and I have } \\
\text { a heart issue." }\end{array}$ \\
\hline Maintain health for family & $\begin{array}{l}\text { Desire to stay in good health for } \\
\text { the sake of one's family }\end{array}$ & $2(13)$ & $\begin{array}{l}\text { "I have a responsibility first to myself and } \\
\text { then to my loved ones to be sure that I'm } \\
\text { taking as good a care of my diabetes as I can." }\end{array}$ \\
\hline $\begin{array}{l}\text { Achieve blood } \\
\text { glucose targets }\end{array}$ & $\begin{array}{l}\text { Desire to maintain target A1c } \\
\text { levels or other blood glucose targets }\end{array}$ & $2(13)$ & \begin{tabular}{l} 
"My goal is 140, I think that's my target goal." \\
\hline
\end{tabular}
\end{tabular}


Table 4 Current practices, self-management behaviors, and compensation techniques $(n=15)$

\begin{tabular}{|c|c|c|c|}
\hline Theme & Definition & $n(\%)$ & Quote \\
\hline $\begin{array}{l}\text { Rely on pump, CGM, } \\
\text { bolus wizard }\end{array}$ & $\begin{array}{l}\text { Trust on technology, i.e., confidence in } \\
\text { pump bolus wizard. }\end{array}$ & 13(87) & $\begin{array}{l}\text { "Whatever the pump is telling me to do is } \\
\text { what I do" }\end{array}$ \\
\hline $\begin{array}{l}\text { Self-management } \\
\text { techniques } \\
\text { for exercise }\end{array}$ & $\begin{array}{l}\text { Comments on self-management } \\
\text { techniques used for exercise. }\end{array}$ & $12(80)$ & $\begin{array}{l}\text { "I check my continuous monitor and if I } \\
\text { know that l'm expending more energy } \\
\text { going up hills or you know working harder } \\
\text { or going faster, I'll go ahead and lower my } \\
\text { temporary basal." }\end{array}$ \\
\hline $\begin{array}{l}\text { Consider trend arrow } \\
\text { directions when bolusing }\end{array}$ & $\begin{array}{l}\text { Comments on use of trend arrows (i.e., if } \\
\text { participants look at trend arrows or allow } \\
\text { trend arrows to influence bolusing } \\
\text { decisions). }\end{array}$ & $11(73)$ & $\begin{array}{l}\text { "I utilize the arrows to see what my blood } \\
\text { sugar is doing, but not necessarily always } \\
\text { make treatment decisions based off the } \\
\text { arrows." }\end{array}$ \\
\hline $\begin{array}{l}\text { Assess food volume, } \\
\text { content, and fat }\end{array}$ & $\begin{array}{l}\text { Confidence in judging food contents and } \\
\text { counting grams of carbohydrates or fat. } \\
\text { Use of techniques such as weighing food or } \\
\text { reading labels. Mentions of habits used to } \\
\text { assist in assessing food content. }\end{array}$ & $10(67)$ & $\begin{array}{l}\text { "I weigh everything I eat. I mean if I eat a } \\
\text { few chips, I put them on a plate on scales } \\
\text { and I weight out how many grams." }\end{array}$ \\
\hline Rely on support network & $\begin{array}{l}\text { Ability to rely on family, friends, clinicians, } \\
\text { etc. to check up on the participant, watch } \\
\text { for blood glucose lows, or help with } \\
\text { management. Also includes participation } \\
\text { in support groups or other educational } \\
\text { events for patients with type } 1 \text { diabetes. }\end{array}$ & $8(53)$ & $\begin{array}{l}\text { "I let her [family member] know that I } \\
\text { arrived, text her. I let her know that I'm } \\
\text { leaving to go someplace and I'm coming } \\
\text { back" }\end{array}$ \\
\hline $\begin{array}{l}\text { Continual adjustment } \\
\text { and monitoring }\end{array}$ & $\begin{array}{l}\text { Continually monitoring blood glucose } \\
\text { levels and adjusting depending on } \\
\text { real-time feedback of blood glucose levels. }\end{array}$ & $8(53)$ & "It's a trial and error thing." \\
\hline $\begin{array}{l}\text { Self-management } \\
\text { techniques } \\
\text { for fat content }\end{array}$ & $\begin{array}{l}\text { Comments on self-management techni- } \\
\text { ques used for meals/meals with high fat } \\
\text { content. }\end{array}$ & $8(53)$ & $\begin{array}{l}\text { "I typically tend to bolus in a dual wave or } \\
\text { just, I monitor my blood sugar so that I } \\
\text { know kind of when that fat hits my system, } \\
\text { then it's time to get some more insulin." }\end{array}$ \\
\hline $\begin{array}{l}\text { Self-management } \\
\text { techniques for alcohol }\end{array}$ & $\begin{array}{l}\text { Comments on self-management techni- } \\
\text { ques used when drinking alcohol. }\end{array}$ & $5(33)$ & $\begin{array}{l}\text { "...if I have a beer or something then I'll } \\
\text { look it up and see how many carbs it is, and } \\
\text { usually I have bolus for twice the amount." }\end{array}$ \\
\hline $\begin{array}{l}\text { Manipulate technology to } \\
\text { achieve desired results }\end{array}$ & $\begin{array}{l}\text { Workarounds used to make technological } \\
\text { tools function as desired; for example, } \\
\text { entering a falsely high number of } \\
\text { carbohydrates that are not actually being } \\
\text { consumed to make pump give larger bolus. }\end{array}$ & $4(27)$ & $\begin{array}{l}\text { “...you exaggerate carbs to get more } \\
\text { insulin.” }\end{array}$ \\
\hline
\end{tabular}

Abbreviation: CGM, continuous glucose monitor.

support network in his diabetes self-management. Participants mentioned the importance of continual adjustment and monitoring of BG to make self-management decisions. There were variations in the type of SMBs used to account for a meal's fat content, including: bolus before meal, use of square or dual wave bolus, monitor BG, and increase a temporary basal rate. Five of the twelve participants who indicated drinking alcohol mentioned various SMBs, including bolusing based on carbohydrate content of the drink, pairing drinks with food, and eating after drinking to avoid lows. Participants also mentioned manipulating the CGM and the pump to achieve desired results, including getting more insulin (670G users). ${ }^{27}$

With respect to future SMBs and plans ( - Table 5) discussed during the interview, there was in general a lack of knowledge on how to account for fat content and compensate for it by changing the bolus waveform. Eleven participants indicated a desire to consider the fat content of food and consider use of an extended wave bolus. With respect to exercise compensation techniques, there was variation between participants, including: changing the timing of the exercise to 2 hours after a meal, eating before exercising, setting up a lower temporal basal or suspending the pump during exercise. Eleven participants stated they wanted to improve exercise compensation. In terms of trend arrows direction, most participants acknowledged lack of knowledge and the need to incorporate that new SMB technique. Only one of the seven participants who indicated consuming alcohol mentioned planning to change alcohol compensation SMBs.

\section{Discussion}

By combining self-tracked exercise, meal, and alcohol events with data from diabetes technology and developing temporally sensitive SMB phenotyping algorithms, we were able to expand upon the typical SMBs analyzed in other adherence studies. $^{5,9,10}$ Phenotyping behaviors within 30 minutes of 
Table 5 Future behavior change plans $(n=15)$

\begin{tabular}{|c|c|c|c|}
\hline Theme & Definition & $n(\%)$ & Quote \\
\hline $\begin{array}{l}\text { Fat content and } \\
\text { insulin dosing }\end{array}$ & $\begin{array}{l}\text { Better estimate meal's fat content when } \\
\text { compensating for meals and if needed } \\
\text { change insulin waveform to square or dual. }\end{array}$ & $11(73)$ & $\begin{array}{l}\text { “...Pay attention to how much fat is in my } \\
\text { food and adjust my dual wave based on that. } \\
\text { Because I've just started kind of dabbling in } \\
\text { that more and I think that that's something } \\
\text { that I can improve upon to help prevent } \\
\text { hyperglycemia." }\end{array}$ \\
\hline $\begin{array}{l}\text { Exercise } \\
\text { compensation }\end{array}$ & $\begin{array}{l}\text { Compensate for exercise (check BG, consume } \\
\text { carbs, and/or deliver insulin, stop or change } \\
\text { basal setting) within } 30 \text { min. }\end{array}$ & $11(73)$ & $\begin{array}{l}\text { "How I'm adjusting my insulin based off of } \\
\text { exercise is something that I'm going to } \\
\text { actively try to improve." }\end{array}$ \\
\hline $\begin{array}{l}\text { Trend arrow } \\
\text { direction }\end{array}$ & $\begin{array}{l}\text { Consider trend arrow direction when } \\
\text { blousing. }\end{array}$ & $8(53)$ & $\begin{array}{l}\text { "I think watching the trend arrows then trying } \\
\text { to account for that with the insulin that I gave } \\
\text { myself is probably a big one." }\end{array}$ \\
\hline $\begin{array}{l}\text { Alcohol } \\
\text { compensation }\end{array}$ & $\begin{array}{l}\text { Compensate for alcohol (check BG, consume } \\
\text { carbs, and/or deliver insulin) within } 30 \text { min. }\end{array}$ & $1(7)$ & $\begin{array}{l}\text { "When I actually have an alcoholic beverage } \\
\text { regardless of what it is [. . .] I really need to } \\
\text { pay attention to that. And not just during but } \\
\text { before and several hours after." }\end{array}$ \\
\hline
\end{tabular}

Abbreviation: BG, blood glucose.

events of interest (Alc30, Exer30, and Meal30) along with examples of meals and alcohol (AlcExmp and MealExmp) are novel in that they previously had not been assessed with objective means or their analyses were not feasible with established methods. Additionally, interactions with the insulin pump (ChgW and Bol10) and hypoglycemia (HypoFreq) were presented in a different fashion than what is typical for a vendor report.

The outcomes from the SMB analysis demonstrated variability of SMBs across the cohort, which is consistent with studies that have evaluated SMBs in youth and children with T1DM using CSII. ${ }^{10,28}$ Our previous work in T1DM patients on CSII therapy using both data from a survey in conjunction with self-tracked data collection using a mHealth app indicated a high intra- and interpatient variability of SMBs. ${ }^{19,20,22,24}$

Findings on variability of SMBs from the phenotyping algorithms used were discussed in follow-up personalized interventions. Our interviews asked about SMBs and practices discovered in the created SMB reports to manage diabetes when eating, drinking alcohol, and exercising. During the interview, participants provided rationale behind those behaviors and were able to identify SMBs that they were interested in changing/improving. Accordant with previous studies, there were considerable variations in SMBs chosen by participants to compensate for alcohol and exercise. , 19,20 $^{2}$

During the personalized educational interventions, participants indicated interest in changing and/or improving SMBs related to fat content of meals. Participant-generated data showed either avoidance of changing the insulin bolus waveform from normal to dual/square, or doing so regularly. The dual/square waveform delivery function was well received by participants who warranted the discussion due to identification of consumption of high fat meals. However, due to the closed-loop system of the MiniMed 670G insulin pump, extra steps are required to change the bolus waveform, thus making this strategy device and behavior specific. These findings all reinforce the need for making temporal, behavioral, and device information available to health providers, as it may prompt them to refer to patient-specific diabetes self-management education or otherwise counsel them about personalized optimal strategies.

Consistent with previous studies, our research identified common barriers to obtaining desirable glycemic control that can inform development of effective interventions. ${ }^{29-33}$ New self-management barriers that emerged from our interviews included the perception of the individual as a medical outlier for which diabetes self-management rules do not always apply. Also, individuals mentioned their personal limitations including barriers to healthy living habits and unpredictable variations. Despite being aware of recommended SMBs, individuals admitted that they can forget to comply on busy days due to the inconvenience of diabetes demands. ${ }^{32,33}$

Concordant with what was reported in study by Grando et al and Patton et al, 27,32 diabetes technology was discussed in both positive and negative manner. Distrust with technology and dissatisfaction with available tools was identified as a barrier. Despite the progression of technology over the years, multiple participants perceived technology as a considerable hindrance to improving glycemic control. Labor and time-intensive manual workarounds to achieve desirable BG levels were described. Diabetes technology as a reliable and useful self-management tool was also discussed. Participants expressed satisfaction with new technologies, including CSII and CGM.

Our study focused directly on SMBs and their temporal relatedness to lifestyle preferences that may require adaptations and were not analyzed for their effects on glycemic control. One of the limitations of this pilot study was the restriction to one CSII device manufacturer and the required use of CGMS. While companies like Glooko and Tidepool provide an environment for integrating data from multiple devices, at the time we began our study, neither platform provided support for the Medtronic 670G. ${ }^{34,35}$ Device restriction simplified the data collection, collation, and analysis and 
provided a more complete dataset to understand behaviors. SMB phenotyping algorithms and infrastructure for assimilating data from various diabetes devices will need to be longitudinally maintained as diabetes technology continues to evolve, thereby creating a moving target for investigators and health providers.

Our cohort came from a single center, had well-controlled diabetes, and was not demographically diverse, which limits the generalizability of the specific behavioral findings. However, the informatics approach to obtaining and presenting this information remains pertinent and applicable to all settings and populations using such devices.

Additionally, while portions of the data analysis and report building were done manually, we anticipate more automation in the future. The next version of the iDECIDE mHealth app includes integration with Apple's HealthKit framework, which has the ability to connect with MyChart (patient portal of the most commonly used electronic health record system in the United States), in near real-time. ${ }^{36,37}$ In addition, behavioral interventions will be delivered over longer periods of time, with multiple educator visits.

The proposed behavioral reports were evaluated by the same research team that was involved in their design. Further evaluation is needed from health providers outside the research team. On other hand, the interviews provided valuable insights into the unique challenges that people living with T1DM face every day and helped to identify knowledge gaps and potential opportunities for positive behavior change.

While patients with chronic illnesses are motivated to use temporal health information from personal data sources to identify trends and optimal timing for interventions, ${ }^{38}$ they may also feel that self-tracking is burdensome, ${ }^{39}$ and mHealth interventions may not keep patients engaged in the long term. ${ }^{40}$ At this stage of research, our vision for the self-tracking module of the iDECIDE app does not entail longterm use by patients in clinical settings, but rather we envision it being used for short periods of time (e.g., 3-4 weeks) during clinical studies or in clinical settings to gather data for measuring baseline or changes of SMBs by populating the proposed SMB report template.

Despite its limitations, we believe that the preliminary findings of this research could help to guide the future design, development, and execution of data-driven personalized diabetes education protocols and tools for patients on insulin pump therapy.

\section{Conclusion}

Optimal diabetes care is predicated on obtaining, interpreting, and incorporating disease-management information into real-time clinical decision making. We developed temporally sensitive algorithms to automatically execute and examine patient-provided information recorded with a mHealth app and objective data obtained from CSII and CGMS. Using this approach, we were able to capture a snapshot of an individual's self-care profile and identify key SMBs and describe them on a sufficiently granular level to inform clinical inference and decision making. We also conducted a thematic analysis of the data-driven educational intervention delivered by a diabetes educator to the participants and identified various themes related to SMBs. Both qualitative and quantitative information demonstrated variability of SMBs across the cohort and thematic analysis of interventions revealed rationale behind the behaviors.

This study describes the first stages of our work and lessons learned to guide the future development of personalized diabetes education protocols and tools. There are plans to further refine the reports and the personalized educational interventions for a larger patient sample.

\section{Clinical Relevance Statement}

Temporally sensitive phenotyping algorithms can be used to populate personalized self-management behavioral reports intended for health providers. Health providers can share the personalized report with patients to guide a data-driven educational intervention and support shared-decision making.

\section{Protection of Human and Animal Subjects}

This study was reviewed by the Mayo Clinic Arizona and Arizona State University Institutional Review Boards.

\section{Conflict of Interest}

None declaared.

\section{References}

1 American Diabetes Association. 8. Pharmacologic approaches to glycemic treatment: standards of medical care in diabetes 2018 . Diabetes Care 2018;41(Suppl 1):S73-S85

2 Rhee MK, Slocum W, Ziemer DC, et al. Patient adherence improves glycemic control. Diabetes Educ 2005;31(02):240-250

3 Nathan DM, Genuth S, Lachin J, et al; Diabetes Control and Complications Trial Research Group. The effect of intensive treatment of diabetes on the development and progression of long-term complications in insulin-dependent diabetes mellitus. N Engl J Med 1993;329(14):977-986

4 Geller AI, Shehab N, Lovegrove MC, et al. National estimates of insulin-related hypoglycemia and errors leading to emergency department visits and hospitalizations. JAMA Intern Med 2014; 174(05):678-686

5 Hendricks M, Monaghan M, Soutor S, Chen R, Holmes CS. A profile of self-care behaviors in emerging adults with type 1 diabetes. Diabetes Educ 2013;39(02):195-203

6 Guilfoyle SM, Crimmins NA, Hood KK. Blood glucose monitoring and glycemic control in adolescents with type 1 diabetes: meter downloads versus self-report. Pediatr Diabetes 2011;12(06): 560-566

7 Pettus J, Price DA, Edelman SV. How patients with type 1 diabetes translate continuous glucose monitoring data into diabetes management decisions. Endocr Pract 2015;21(06):613-620

8 Beverly EA, Ganda OP, Ritholz MD, et al. Look who's (not) talking: diabetic patients' willingness to discuss self-care with physicians. Diabetes Care 2012;35(07):1466-1472

9 Driscoll KA, Wang Y, Bennett Johnson S, et al. White coat adherence in pediatric patients with type 1 diabetes who use insulin pumps. J Diabetes Sci Technol 2016;10(03):724-729

10 Driscoll KA, Johnson SB, Hogan J, Gill E, Wright N, Deeb LC. Insulin bolusing software: the potential to optimize health outcomes in type 1 diabetes mellitus. J Diabetes Sci Technol 2013;7(03):646-652 
11 O'Connell MA, Donath S, Cameron FJ. Poor adherence to integral daily tasks limits the efficacy of CSII in youth. Pediatr Diabetes 2011;12(06):556-559

12 Patton SR, Clements MA, Fridlington A, Cohoon C, Turpin AL, Delurgio SA. Frequency of mealtime insulin bolus as a proxy measure of adherence for children and youths with type 1 diabetes mellitus. Diabetes Technol Ther 2013;15(02):124-128

13 Carlson AL, Mullen DM, Bergenstal RM. Clinical use of continuous glucose monitoring in adults with type 2 diabetes. Diabetes Technol Ther 2017;19(S2):S4-S11

14 Haviland N, Walsh J, Roberts R, Bailey TS. Update on clinical utility of continuous glucose monitoring in type 1 diabetes. Curr Diab Rep 2016;16(11):115

15 Tao D, Yuan J, Qu X. Presenting self-monitoring test results for consumers: the effects of graphical formats and age. J Am Med Inform Assoc 2018;25(08):1036-1046

16 Gandhi K, Vu BK, Eshtehardi SS, Wasserman RM, Hilliard ME. Adherence in adolescents with type 1 diabetes: strategies and considerations for assessment in research and practice. Diabetes Manag (Lond) 2015;5(06):485-498

17 Ziegler R, Rees C, Jacobs N, et al. Frequent use of an automated bolus advisor improves glycemic control in pediatric patients treated with insulin pump therapy: results of the Bolus Advisor Benefit Evaluation (BABE) study. Pediatr Diabetes 2016;17(05):311-318

18 Herbert LJ, Sweenie R, Kelly KP, Holmes C, Streisand R. Using qualitative methods to evaluate a family behavioral intervention for type 1 diabetes. J Pediatr Health Care 2014;28(05):376-385

19 Grando MA, Groat D, Soni H, et al. Characterization of exercise and alcohol self-management behaviors of type 1 diabetes patients on insulin pump therapy. J Diabetes Sci Technol 2017;11(02):240-246

20 Groat D, Grando MA, Soni H, et al. Self-management behaviors in adults on insulin pump therapy. J Diabetes Sci Technol 2017;11 (02):233-239

21 Groat D, Soni H, Grando MA, Thompson B, Cook CB. Self-reported compensation techniques for carbohydrate, exercise, and alcohol behaviors in patients with type 1 diabetes on insulin pump therapy. J Diabetes Sci Technol 2018;12(02):412-414

22 Karway G, Grando MA, Grimm K, Groat D, Cook CB, Thompson B. Selfmanagement behaviors of patients with type 1 diabetes: comparing two sources of patient-generated data. Appl Clin Inform 2020;11 (01):70-78

23 Montori VM, Gafni A, Charles C. A shared treatment decision-making approach between patients with chronic conditions and their clinicians: the case of diabetes. Health Expect 2006;9(01):25-36

24 Groat D, Soni H, Grando MA, Thompson B, Kaufman D, Cook CB. Design and testing of a smartphone application for real-time selftracking diabetes self-management behaviors. Appl Clin Inform 2018;9(02):440-449
25 Centers for Disease Control and Prevention. Measuring physical activity intensity [Internet]. CDC 24/7: saving lives, protecting people; 2015. Available at: https://www.cdc.gov/physicalactivity/basics/measuring/index.html. Accessed June 7, 2018

26 Braun V, Clarke V. Using thematic analysis in psychology. Qual Res Psychol 2006;3(02):77-101

27 Grando MA, Bayuk M, Karway G, et al. Patient perception and satisfaction with insulin pump system: pilot user experience survey. J Diabetes Sci Technol 2019;13(06):1142-1148

28 Patton SR, Driscoll KA, Clements MA. Adherence to insulin pump behaviors in young children with type 1 diabetes mellitus. J Diabetes Sci Technol 2017;11(01):87-91

29 Martyn-Nemeth P, Duffecy J, Fritschi C, Quinn L. Challenges imposed by hypoglycemia in adults with type 1 diabetes. Clin Nurs Res 2019;28(08):947-967

30 Laranjo L, Neves AL, Costa A, Ribeiro RT, Couto L, Sá AB. Facilitators, barriers and expectations in the self-management of type 2 diabetes-a qualitative study from Portugal. Eur J Gen Pract 2015; 21(02):103-110

31 Ritholz MD, Beverly EA, Weinger K. Digging deeper: the role of qualitative research in behavioral diabetes. Curr Diab Rep 2011; 11(06):494-502

32 Abdoli S, Hardy LR, Hall J. The complexities of "Struggling to Live Life". Diabetes Educ 2017;43(02):206-215

33 Kent DA, Quinn L. Factors that affect quality of life in young adults with type 1 diabetes. Diabetes Educ 2018;44(06):501-509

34 Glooko, Inc. Glooko [Internet]. Diabetes remote monitoring. 2018. Available at: https://www.glooko.com/. Accessed January 29, 2018

35 Tidepool. Tidepool [Internet]. Liberate your data. Available at: https://tidepool.org/. Accessed January 30, 2018

36 Epic Systems Corporation. Epic [Internet]. Available at: https:// www.epic.com/. Accessed October 2, 2018

37 Kumar RB, Goren ND, Stark DE, Wall DP, Longhurst CA. Automated integration of continuous glucose monitor data in the electronic health record using consumer technology. J Am Med Inform Assoc 2016;23(03):532-537

38 Barbarin AM, Klasnja P, Veinot TC. Good or bad, ups and downs, and getting better: use of personal health data for temporal reflection in chronic illness. Int J Med Inform 2016; 94:237-245

39 Ancker JS, Witteman HO, Hafeez B, Provencher T, Van de Graaf M, Wei E. "You Get Reminded You're a Sick Person": personal data tracking and patients with multiple chronic conditions. J Med Internet Res 2015;17(08):e202

40 Nelson LA, Coston TD, Cherrington AL, Osborn CY. Patterns of user engagement with mobile- and web-delivered self-care interventions for adults with T2DM: a review of the literature. Curr Diab Rep 2016;16(07):66 